\title{
Estudo da degradação de compostos fenólicos presente em águas residuárias de postos de combustíveis utilizando fungos filamentosos (Aspergillus Flavus)
}

Degradation study of phenolic compounds in waste water of gas stations using filamentous fungi (Aspergillus Flavus)

\author{
Evandro Zanin', lara Elisa Cardoso da Silva², Toni Jefferson Lopes³, \\ Adriano Cancelier ${ }^{4}$, Murilo Cesar Costeli ${ }^{5}$, Adriano da Silva ${ }^{6}$ \\ 1,2,5 Universidade Comunitária da Região de Chapecó, Chapecó, SC, Brasil \\ 3,6 Universidade Federal do Rio Grande, Santo Antônio da Patrulha, RS, Brasil \\ ${ }^{4}$ Universidade Federal de Santa Maria, Santa Maria, RS, Brasil
}

\begin{abstract}
Resumo
Diversos compostos são sintetizados e produzidos industrialmente, onde, em sua maioria, são dificilmente degradados ou reciclados na forma em que se encontram. Grande parcela do processo de contaminação pode ser atribuída aos compostos fenólicos, encontrados nas mais diversas concentrações em águas residuárias de postos de combustíveis. Neste estudo, buscou-se isolar e identificar fungos filamentosos com capacidade de degradação de compostos fenólicos e avaliar, através da utilização de técnicas de planejamento experimental, os fatores: temperatura e pH no rendimento da degradação dos compostos fenólicos. A linhagem do fungo isolada foi a de Asperillus flavus. Através dos resultados obtidos verificou-se que a linhagem de fungos estudada é pouco afetada por variações no $\mathrm{pH}$ e apresentou melhor desempenho na temperatura de $25^{\circ} \mathrm{C}$ e pH 8, com degradação de 6,7 ppm de fenol.
\end{abstract}

Palavras-chave: Fungos filamentosos, Compostos fenólicos, Tratamento de efluentes, Planejamento experimental.

\begin{abstract}
Several compounds are synthesized and industrially produced, where in most cases, are hardly degraded or recycled in the way they are. A large portion of contamination process can be attributed to phenolic compounds found with different concentrations in wastewater from gas stations. In this study, we attempted to isolate and identify filamentous fungi capable of phenolic compounds degradation and evaluate, using experimental design techniques, the effects of temperature and $\mathrm{pH}$ on degradation yield of phenolic compounds. Aspergillus flavus fungi lineage was isolated in this study. Results showed that the fungi lineage was little affected by changes in $\mathrm{pH}$ and showed better performance at $25^{\circ} \mathrm{C}$ and $\mathrm{pH} 8$, when $6.7 \mathrm{ppm}$ of phenol was degraded.
\end{abstract}

Keywords: Filamentous fungi, Phenolic compounds, Effluent treatment, Experimental design . 


\section{INTRODUÇÃO}

A contaminação das águas com o aumento das atividades industriais, juntamente com os resíduos introduzidos no ambiente estão provocando a degradação dos ecossistemas e dos recursos naturais disponíveis para o homem, onde muitas dessas substâncias são de natureza persistente, como exemplo, os compostos fenólicos.

O acúmulo de insumos e a ineficiência dos processos de conversão dos resíduos industriais são os principais fatores de contaminação do ambiente. Nesse contexto, busca-se o mais adequado tratamento destes resíduos químicos em função da grande diversidade e complexidade de efluentes, aliado às imposições da legislação que exigem tratamentos eficientes.

Uma grande parcela dos processos de contaminação química pode ser atribuída, principalmente, aos compostos fenólicos encontrados em águas residuárias das empresas, como refinarias de petróleo, indústrias químicas, têxteis, papel e celulose, além de postos de combustíveis e oficinas mecânicas entre outras.

Os compostos fenólicos enquadram-se nos resíduos resultantes da biodegradação de resíduos naturais (VASSILEV et al., 1994) e da atividade antrópica; são encontrados no solo e na água, mas apesar de distribuídos na natureza fazem parte dos principais poluentes tóxicos residuais descartados por uma grande variedade de indústrias (MARR et al., 1996; KARAM \& NICELL, 1997; PIERREHUMBERT et al., 2002). São reconhecidos como poluentes prioritários por apresentarem grande toxidade e serem dificilmente degradados ou reciclados, sendo que alguns de seus subprodutos, como clorofenóis e cresóis são designados como poluentes primários, ou seja, originados diretamente das fontes de emissão.

Devido a sua alta volatilidade e solubilidade em água, os fenóis conferem problemas de gosto e odor em águas potáveis, além da toxidade. A concentração de $0,5 \mathrm{mg} \mathrm{L}-1$ de fenóis foi estabelecida como padrão de lançamento para qualquer tipo de efluente, de acordo com a resolução do CONAMA, sendo que, no Brasil, em águas destinadas ao abastecimento, o Ministério da Saúde determinou, a partir da portaria 1469, que o limite máximo permitido de fenol seja de $0,1 \mu \mathrm{g} \mathrm{L}-1$, a fim de evitar danos à saúde humana. Em uma portaria mais recente do Ministério da Saúde, Portaria n 518 (2004), são estipuladas, em águas de abastecimento, concentrações máximas apenas para compostos derivados do fenol, tais como pentaclorafenol (9 $\mu \mathrm{g} \mathrm{L}-1)$ e 2,4,6 triclorofenol (0,2 $\mu \mathrm{g} \mathrm{L}-1)$.

Na solução deste problema muitas alternativas já são utilizadas para a diminuição aos níveis aceitáveis da legislação. A utilização de microrganismos nativos ou introduzidos no ambiente, ou mesmo a captação e disposição do resíduo nocivo em locais de tratamento, biopilha, lodo ativado ou outros reatores biológicos são realizados com sucesso em lagoas de tratamento de águas residuárias em diversas indústrias.

Fungos e bactérias são os principais agentes decompositores presentes na natureza. Contudo, para as bactérias, os compostos fenólicos com ação recalcitrante possuem ação bactericida, inibindo esse tipo de tratamento biológico. Já os fungos são microrganismos mais resistentes a várias condições ambientais adversas, como variações de $\mathrm{pH}$, luz, umidade e oxigênio (SANTANELLA, 1996; OLIVEIRA, 2005), além de ter a capacidade de produzir inúmeras enzimas como lactase, proteases, ligninases, lípases, celulases, fenoloxidases, peroxidases entre outras utilizadas para auxiliar a sua nutrição (BENNET, 1998), atuando como agentes deteriorantes para facilitar a biodegradação pelo fungo, sendo esta degradação de alto potencial, além do potencial biossortivo (metais pesados e corantes).

O presente trabalho tem como objetivo avaliar as melhores condições de degradação de compostos fenólicos em processo estático, utilizando fungos filamentosos (Aspergillus flavus). Para isso, analisou-se a influência de 2 fatores ( $\mathrm{pH}$ e temperatura) sobre a capacidade de degradação dos compostos fenólicos pelo fungo filamentoso selecionado, utilizando a metodologia de planejamento fatorial.

\section{MATERIAL E MÉTODOS}

As amostras de águas residuárias foram coletadas em postos de combustíveis localizados na cidade de Chapecó - SC. Durante a coleta com frascos plásticos, utilizaram-se os procedimentos descritos nas normas NBR 9897/87 e NBR 9898/87, mantendo a proporção entre líquido e o sólido em suspensão. Posteriormente, as amostras foram armazenadas a uma temperatura de $10{ }^{\circ} \mathrm{C}$. 
No isolamento das linhagens de fungos foi utilizado o método de profundidade (pour-plate) para um volume de efluente de $1 \mathrm{~mL}$ (diluição de 10:1) e suas respectivas diluições decimais seriadas até 10-9, em meio de cultura contendo: $15 \mathrm{~g}$ de extrato de malte; $1 \mathrm{~g}$ de dextrose-peptona; $5 \mathrm{~g}$ de ácido gálico; 20 g de Agar; $1 \mathrm{~L}$ de água destilada (DAVIDSON et al., 1983). Após a inoculação, incubou-se as placas contendo o material inoculado a uma temperatura de $28 \pm 2{ }^{\circ} \mathrm{C}$ por sete dias.

A purificação das linhagens de fungos filamentosos foi realizada utilizando o meio de cultura tipo SABOURAUD (MANUAL MERK, 1982), que apresenta em sua composição: $5 \mathrm{~g}$ de extrato de levedura; $5 \mathrm{~g}$ de peptona; $20 \mathrm{~g}$ de sacarose; $10 \mathrm{~g}$ de Agar; $1 \mathrm{~g}$ de ácido gálico; $1 \mathrm{~L}$ de água destilada. Após a inoculação, incubou-se as placas contendo o material inoculado a uma temperatura de $28 \pm 2{ }^{\circ} \mathrm{C}$ por sete dias.

A identificação das linhagens foi realizada através de análises das características morfológicas de cada fungo, foi observada a coloração e a textura superficial da colônia de fungos e as características das hifas, a partir da técnica do microcultivo, seguido de coloração com azul de lactofenol.

O planejamento experimental utilizado foi de 32, considerando a influência de dois fatores ( 3 níveis em cada fator) sobre o rendimento da degradação de compostos fenólicos por fungos filamentosos (Asperillus flavus): A) Temperatura $\left(25,28\right.$ e $\left.30^{\circ} \mathrm{C}\right)$ e $\left.\mathrm{B}\right) \mathrm{pH}(4,6$ e 8$)$. O volume utilizado foi de $200 \mathrm{~mL}$ do efluente bruto filtrado e pasteurizado com uma quantidade fixa de, aproximadamente, 20 $\mathrm{mg}$ de fungos em um erlenmeyer de $250 \mathrm{~mL}$. Realizou-se o experimento em duplicata por 5 dias com concentração inicial de fenol (ácido gálico) de $10 \mathrm{ppm}$. O pH inicial foi medido e ajustado através da utilização de soluções de ácido clorídrico $(\mathrm{HCl})$ de $1,0 \mathrm{M}$ e de hidróxido de sódio $(\mathrm{NaOH})$ de 1,0 M.

Este planejamento foi avaliado com o software Statistica $6.0{ }^{\circledR}$. Um modelo quadrático com interações de $2^{\mathrm{a}}$ ordem foi ajustado sobre os resultados finais.

Os teores de fenóis totais das águas residuárias foram determinados por espectrofotometria, utilizando-se um conjunto de padrões de reagentes para fenol, de acordo com a metodologia da ALFAKIT, através da análise de fenol fotocolorimétrico. As análises foram realizadas a cada intervalo de tempo de $24 \mathrm{~h}$ e os resultados estão expressos em ppm de equivalente de ácido gálico da amostra. A concentração de fenol equivalente de ácido gálico foi determinada por espectrofotometria, de acordo com as diretrizes para obtenção de máxima absorbância, no comprimento de onda de $470 \mathrm{~nm}$ (ALFAKIT, 2009).

\section{RESULTADOS E DISCUSSÃO}

\section{Isolamento e Determinação do Fungo Filamentoso}

Através do isolamento dos microrganismos observou-se que, depois de várias replicações para a seleção dos fungos com melhores qualidades degradativas do fenol, houve a predominância de uma colônia fúngica. Analisando as características, através da placa de microcultivo, foi possível observar que o fungo filamentoso isolado foi o Asperillus flavus, mostrado nas Figuras 1 e 2.

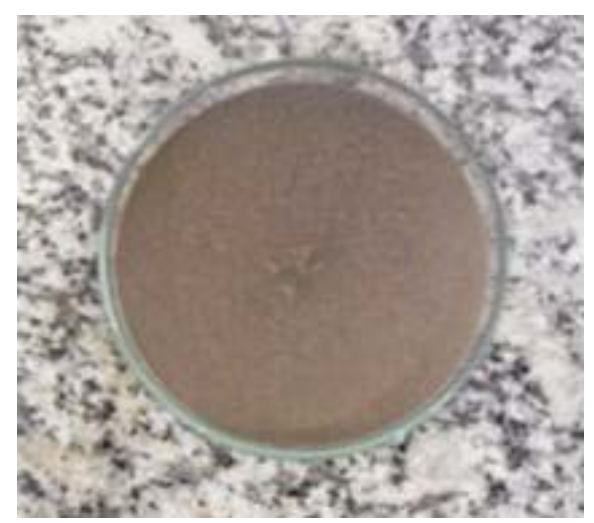

Figura 1- Colônia do fungo: vista frontal.

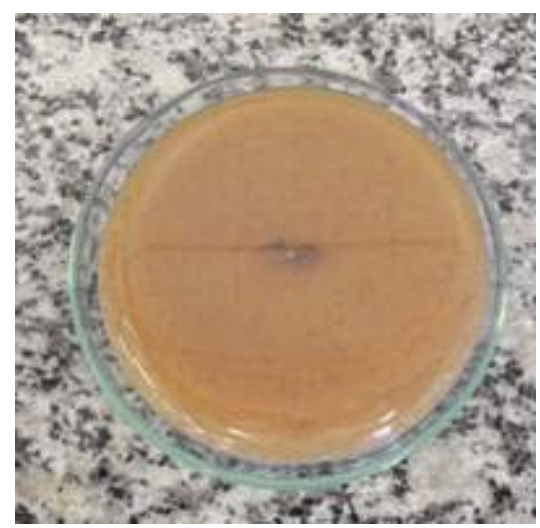

Figura 2 - Colônia do fungo: vista do fundo. 
Nas Figuras 1 e 2 pode-se observar a presença de fungos Aspergillus flavus, os quais apresentam características de um fungo filamentoso com textura cotonosa a lanosa, plana com sulcos radiais e coloração amarelo-esverdeada. O reverso é branco, cinza ou marrom. Um exsudado incolor ou marrom-claro pode ser produzido pela colônia, possuindo uma temperatura máxima de crescimento de $35^{\circ} \mathrm{C}$ (PASSOS et al., 2006).

LEMOS \& ARAUJO (2002) isolaram e identificaram 75 colônias no solo contaminado de Guararema, de onde 60 apresentaram capacidade de degradar hidrocarbonetos de petróleo, de 4 gêneros fúngicos (Aspergillus sp, Penicillium sp, Paecilomyces sp e Fusarium sp). SANTOS \& LINARDI (2004) isolaram 15 linhagem de 4 gêneros (Fusarium sp, Penicillium sp, Aspergillus sp e Graphium sp) e também foi comprovado a eficiências dessas linhagens de fungos filamentosos na degradação de compostos fenólicos em efluentes industriais.

\section{Capacidade de Degradação de Compostos Fenólicos pelos Fungos Filamentosos}

Para obtenção de informações preliminares, foram feitos ensaios de degradação de compostos fenólicos, a determinados valores de $\mathrm{pH}(4,6$ e 8$)$ e de temperatura $\left(25,28\right.$ e $\left.30^{\circ} \mathrm{C}\right)$, como mostra a Figura 3.

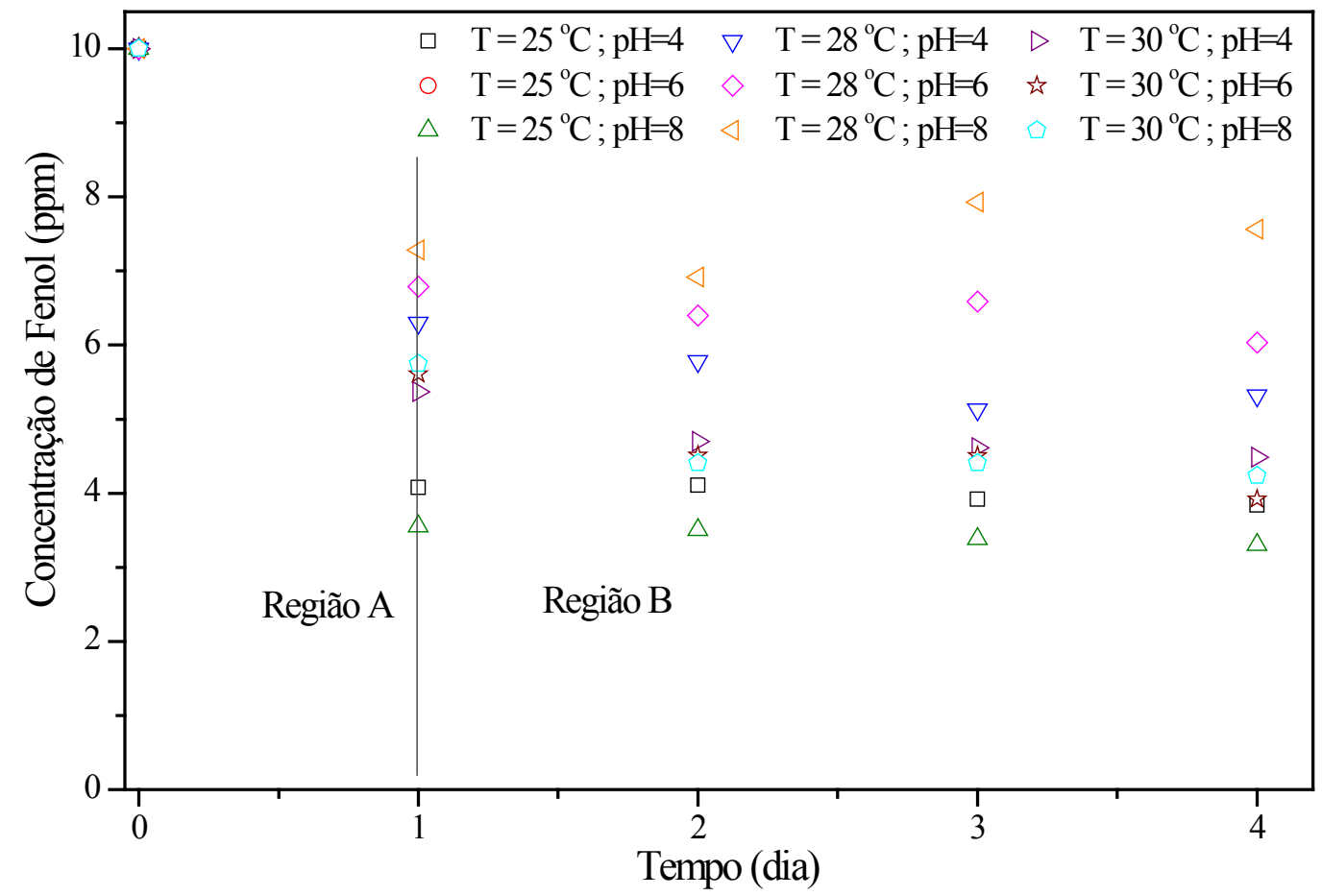

Figura 3 - Comportamento da concentração de fenol ao longo do tempo para diferentes condições de temperatura e pH.

Observa-se, na Figura 3, que a Temperatura de $25^{\circ} \mathrm{C}$, em todas as faixas de $\mathrm{pHs}$ testados, foi onde ocorreu uma acentuada redução dos compostos fenólicos presentes em solução. Ainda, é possível observar duas regiões distintas na Figura 03, destacadas por região A e região B. Na região A ocorre uma degradação acentuada do fenol pelo fungo, num período de 1 dia. Na região $\mathrm{B}$, após o primeiro dia, a concentração das substâncias fenólicas praticamente se estabiliza, nas diversas temperaturas e $\mathrm{pH}$ testados.

Ao longo do primeiro dia (Região A) a concentração dos esporos do fungo era menor, fazendo com que a degradação do fenol fosse mais eficiente, pois havia substrato e nutrientes suficientes para os fungos se multiplicarem. Na Região B, pode-se observar uma degradação de fenol mais lenta, em comparação a Região A, devido à demanda por substrato (fenol) ser menor para uma maior quantidade de fungo, havendo competição entre os esporos do fungo pelo substrato.

A degradação de compostos fenólicos por fungos filamentosos segue um comportamento seme- 
lhante para as 9 (nove) diferentes condições avaliadas, alcançando o equilíbrio aparente em cerca de 2 dias.

\section{FATORES QUE AFETAM O PROCESSO DE DEGRADAÇÃO DE COMPOSTOS FENÓLICOS}

Posteriormente a etapa de isolamento, identificação da espécie de fungo presente nas inoculações e da determinação do comportamento da degradação de compostos fenólicos devido ao pH e a temperatura, foi realizada a avaliação da capacidade do fungo em degradar os compostos fenólicos comumente encontrados em águas residuárias de postos de combustíveis. A matriz experimental adotada, com seus respectivos ensaios experimentais, é mostrada na Tabela 1, sendo que em todos os casos foi utilizada uma concentração inicial de fungo de $0,1 \mathrm{mg} \mathrm{mL}-1$ (Volume de efluente de $200 \mathrm{~mL}$ ).

Tabela 1: Fatores e Níveis estudados para a deterioração de compostos fenólicos por fungos filamentosos (Resposta).

\begin{tabular}{ccc}
\hline $\begin{array}{c}\text { Fator } 1 \\
\mathrm{pH}\end{array}$ & $\begin{array}{c}\text { Fator } 2 \\
\text { Temperatura }\left({ }^{\circ} \mathrm{C}\right)\end{array}$ & $\begin{array}{c}\text { Resposta } \\
\text { Concentração média de Fenol }(\mathrm{ppm})^{*}\end{array}$ \\
\hline 4 & 25 & 6,16 \\
4 & 28 & 4,67 \\
4 & 30 & 5,51 \\
6 & 25 & 6,38 \\
6 & 28 & 3,97 \\
6 & 30 & 6,08 \\
8 & 25 & 6,70 \\
8 & 28 & 2,43 \\
8 & 30 & 5,77 \\
\hline
\end{tabular}

*Biomassa de fenol (concentração equivalente de ácido gálico) degradado.

$\mathrm{Na}$ Tabela 2 são mostrados os valores dos efeitos referentes aos fatores $\mathrm{pH}$ e temperatura e seus respectivos índices estatísticos. Pode-se observar que todos os efeitos são significativos, com exceção do termo linear da temperatura, denotado pelo nível $\mathrm{p}<0,05$.

Tabela 2: Cálculo dos efeitos e respectivos índices estatísticos.

\begin{tabular}{ccccccc}
\hline \multirow{2}{*}{ Fatores } & Efeito & Desvio & & \multicolumn{2}{c}{ Limite de confiança } \\
\cline { 6 - 7 } & & Padrão & $\mathrm{p}$ & $-95 \%$ & $+95 \%$ \\
\hline Média/Interações & 5,3 & 0,117 & 0,000 & 5,016 & 5,618 \\
$(\mathrm{~L})$ Temperatura $\left({ }^{\circ} \mathrm{C}\right)$ & $-0,62$ & 0,286 & 0,0799 & $-1,361$ & 0,108 \\
$(\mathrm{Q})$ Temperatura & $-2,34$ & 0,249 & 0,0002 & $-2,988$ & $-1,706$ \\
$(\mathrm{~L}) \mathrm{pH}$ com $(\mathrm{Q})$ Temperatura & $-1,32$ & 0,303 & 0,0073 & $-2,099$ & $-0,540$ \\
\hline
\end{tabular}

(Q) = Termo Quadrático

$(\mathrm{L})=$ Termo Linear

Os valores de efeito apresentados na Tabela 2 representam as influências dos fatores Temperatura e $\mathrm{pH}$, na variável de processo dependente (Concentração final de fenol). Também, é possível destacar que todos os efeitos apresentaram valores negativos, isto demonstra que a degradação de 
fenol se comporta de forma inversa ao efeito dos fatores, por exemplo, um aumento da temperatura ocasiona uma redução da concentração de fenol.

O modelo empírico obtido para a degradação de fenol pelo fungo Aspergillus flavus é representado pela Equação 1.

$$
\beta=302,96-21,64 \cdot T+0,39 \cdot T^{2}-0,00016 \cdot p H \cdot T^{2}
$$

com coeficiente de determinação $\mathrm{R}^{2}$ igual à 0,9795 , e onde $\beta$ :é a concentração de fenol no final do processo.

A análise de variância do modelo é mostrada na Tabela 3. Observa-se que a soma quadrática dos resíduos é muito pequena, o que nos indica que o modelo é bom. Observando os valores de $\mathrm{F}$ calculados para os resíduos em relação à regressão, vemos que é superior a sete vezes o valor de $\mathrm{F}$ tabelado (BARROS NETO et al., 1996). A regressão é significativa, podendo ser usada para fins de predição. Todos os índices estatísticos mostram que o modelo ajustado descreve bem os resultados experimentais.

Tabela 3: Análise de variância do modelo para a degradação de fenol por fungos filamentosos.

\begin{tabular}{lccccc}
\hline $\begin{array}{l}\text { Fonte de } \\
\text { variação }\end{array}$ & $\begin{array}{c}\text { Soma } \\
\text { Quadrática }(\mathrm{SQ})\end{array}$ & $\begin{array}{c}\text { Graus de } \\
\text { liberdade }(v)\end{array}$ & $\begin{array}{c}\text { Média Quadrática } \\
(\mathrm{MQ})\end{array}$ & $\begin{array}{c}\mathrm{F}_{\mathrm{v} 1, v 2} \\
\text { calculado }\end{array}$ & $\begin{array}{c}\mathrm{F}_{\mathrm{v} 1, v 2} \\
\text { tabelado }\end{array}$ \\
\hline Regressão & 14,52847 & 3 & 4,84282 & 39,46 & 5,41 \\
Resíduos & 0,61353 & 5 & 0,12270 & & \\
Total & 15,14200 & 8 & & & \\
\hline
\end{tabular}

A Figura 4 mostra à superfície de resposta gerada pelo modelo quadrático com interações entre os fatores (Equação 1).

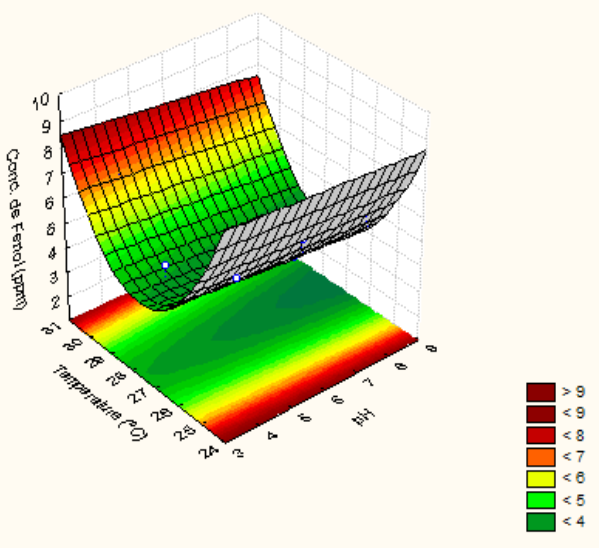

Figura 4 - Superfície de resposta da concentração final de fenol em função da Temperatura e pH.

$\mathrm{Na}$ Figura 4 pode-se observar que o melhor rendimento se encontra no intervalo de temperatura de 25 e $30^{\circ} \mathrm{C}$. Porém, a $30^{\circ} \mathrm{C}$, o fungo Aspergillus flavus possui a sua temperatura máxima de crescimento, não conseguindo atuar de forma satisfatória na degradação dos compostos fenólicos. Outros autores como Silva et al. (2007) encontraram 4 linhagens de fungos filamentosos deteriorantes de compostos fenólicos (Aspergillus flavus, Cladosporium sp, Penicilium sp e Phoma sp), com degradação de 703 mg de ácido gálico em 96 horas para o fungo Aspergillus flavus.

PASSOS et al. (2006), utilizando Aspergillus sp. encapsulados em alginato de sódio, mostraram que o encapsulamento dos fungos proporciona um microambiente mais favorável para o fungo, 
protegendo-o do estresse abiótoco para a biodegradação, atingindo degradações de até 989 mg L-1 de fenol em efluente sintético.

Nos ensaios experimentais para a determinação da degradação de fenol por Aspergillus flavus, também foi realizada a quantificação dos valores da massa de fungo final e da biomassa seca. Os valores de biomassa microbiana foram obtidos após decorridos 4 dias do processo, sendo estes mostrados na Tabela 4 e Figura 5.

Tabela 4: Matriz Experimental tendo como resposta os valores médios de massa de fungo final e de Biomassa Microbiana Seca

\begin{tabular}{ccc}
\hline Ensaio & $\begin{array}{c}\text { Valor médio da massa de } \\
\text { fungo final }(\mathrm{mg})\end{array}$ & $\begin{array}{c}\text { Valor médio da } \\
\text { Biomassa seca }(\mathrm{mg})\end{array}$ \\
\hline 1 & 23,8 & 3,8 \\
2 & 24,1 & 4,1 \\
3 & 24,5 & 4,5 \\
4 & 30,5 & 10,5 \\
5 & 30,6 & 10,6 \\
6 & 27,4 & 7,4 \\
7 & 26,4 & 6,4 \\
8 & 28,6 & 8,6 \\
9 & 35,1 & 15,1 \\
\hline
\end{tabular}

$\mathrm{Na}$ Figura 5, pode-se observar que os maiores valores para a biomassa microbiana estão situados entre $\mathrm{pH}$ 6,0 e 8,0 e esta tendência pode ter provocado uma redução na degradação de fenol, em alguns casos, devido à competição por substrato entre os fungos. $\mathrm{O}$ valor de biomassa seca encontrado para o ensaio com maior degradação de fenol $\left(25^{\circ} \mathrm{C} \mathrm{e} \mathrm{pH} 8,0\right)$ foi de $6,4 \mathrm{mg}$, que no caso foi um valor intermediário entre todos os valores encontrados.

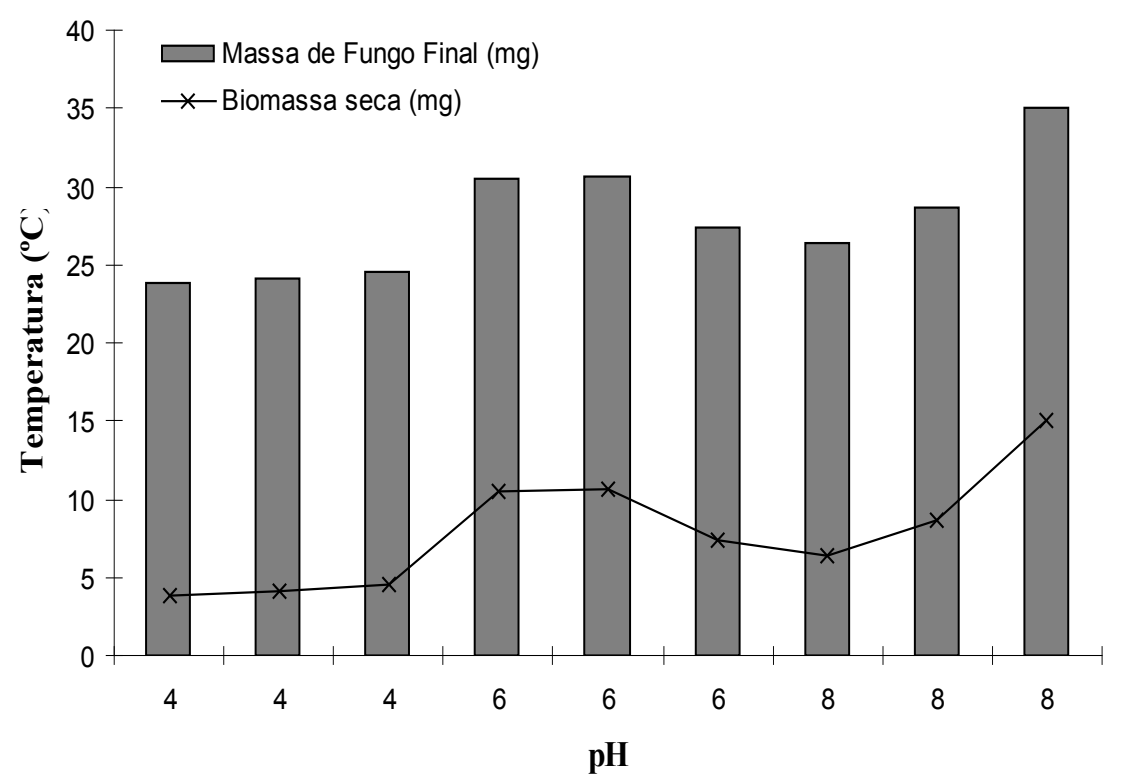

Figura 5 - Variação da quantidade de massa final de fungo e biomassa seca em relação ao pH e Temperatura. 


\section{CONCLUSÕES}

Foi possível isolar e purificar uma linhagem de fungo filamentoso presente em águas residuárias de postos de gasolina. A espécie isolada foi Aspergillus flavus, onde, experimentalmente, observou-se sua capacidade em degradar compostos fenólicos presentes nas águas residuárias de postos de combustíveis.

Através da análise estatística verificou-se que o rendimento na degradação do fenol deste estudo é influenciado, principalmente, pela temperatura. Também, especula-se a influência do aumento da concentração dos fungos pela combinação do efeito do aumento de Temperatura e de $\mathrm{pH}$ em conjunto. Os melhores resultados foram encontrados a $25^{\circ} \mathrm{C}$ e pH 8 , com deterioração de 6,7 ppm de ácido gálico e 6,4 mg de biomassa seca.

\section{REFERÊNCIAS}

ALFAKIT Ltda Catálogo Técnico, Florianópolis, SC, 2009.

BARROS NETO, B.; SCARMINIO, I. S.; BRUNS, R. E. Planejamento Experimental e Otimização de Experimentos, 2 ed, Campinas:Editora Unicamp, 299 p, 1996.

BENNET, J.W. Mycotechnology: the role of fungi in biotechnology. Journal of Biotechnology, v.66, p.101107, 1998.

CONAMA Resolução CONAMA no 357, de 17 de março de 2005.

Davidson, R.W.; Campbell, W.A.; Blaisdell, D.J. Differentiation of wood-decaying fungi by their reactions on gallic or tannic acid medium. Journal of Agriculture Resource, v.57, n.9, p.683-685, 1983.

KARAN, J.; NICELL, J.A. Potential applications of enzymes in wastes treatment. Journal of Chemical Technology and Biotechnology, v.69, p.141-153, 1997.

LEMOS, J. L. S.; ARAUJO, F. M. S. Isolamento e identificação de fungos degradadores de petróleo. In: X Jornada de Iniciação científica do CETEM, Rio de Janeiro, 2002.

MANUAL de meios de cultivo MERCK. Rio de Janeiro: Química, 189 p, 1982.

MARR, J.; K REMER, S.; STERNER, O.; ANKE, H. Transformation and mineralization of halophenols by Penicillium simplicissimum SK9117. Biodegradation, v.7, p.165-171, 1996.

MINISTÉRIO DA SAÚDE Portaria 36/GM-MS. Disponível em: www.mp.sp.gov.br/portal/page/portal/cao_ consumidor/legislacao/leg_servicos_publico/leg_sp_agua/Port36-90GM-MS. Acesso em 12 de janeiro de 2009.

MINISTÉRIO DA SAÚDE Portaria GM Nº 518 de 2004. Disponível em: http://dtr2001.saude.gov.br/sas/ PORTARIAS/Port2004/GM/GM-518.htm. Acesso em 12 de janeiro de 2009.

OLIVEIRA, C. E.; FÉLIX, J. P. L.; LEITÃO, C. R. Degradação de fenóis presentes em águas residuárias de refinarias de petróleo. In: $23^{\circ}$ Congresso Brasileiro de Engenharia Sanitária e Ambiental, Associação Brasileira de Engenharia Sanitária e Ambiental (ABES), Campo Grande/MS, v.1, p. 1-8, 2005.

PASSOS, C. T. BURKET, C. A. V; KALIL, S. J. Estudo da biodegradação do fenol por uma nova linhagem de Aspergillus sp. Rio Grande, RS, 84p. Dissertação (Mestrado em Engenharia e Ciência de Alimentos, FURG, 2006.

PIERREHUMBERT, G.; DROZ, P. O.; TARDIF, R.; TARDIF, G. C.; TRUCHON, G. RENGARAJ, S.; 
MOON, S-H.; SIVABALAN, S.; ARABINDOO, B.; MURUGESAN, V. Removal of phenol from aqueous solution and resin manufacturing industry wastewater using an agricultural waste: rubber seed coat. Journal of Hazardous Materials B., v.89, p.185-196, 2002.

SANTAELLA, S. T. Avaliação da eficiência da estação de tratamento de esgotos de uma indústria de beneficiamento de castanha de caju. Relatório de Pesquisa. Departamento de Engenharia Hidráulica e Ambiental, Universidade Federal do Ceará, Fortaleza, Brasil, 1996.

SANTOS, V. L.; LINARDI, V. R. Biodegradaton of phenol by a filamentous fungi isolated from industrial effluents - identification and degradation potential. Process Biochemistry. n.39, p.1001-1006, 2004.

SILVA , I.E.C. ; LUDWIG, K.V.F.; NEUMANN, D.; SCHNEIDER, A.C.; ONOFRE, S.B. Fungos filamentosos degradadores de compostos fenólicos isolados de águas residuárias de postos de combustíveis. Revista de Biologia e Saúde da UNISEP. v.1, n.1, 2, p.101-108, 2007.

VASSILEV, N.; BACA, M.T.; VASSILEVA, M. Plant lignocellulose and decomposition by fungy: from nature to industrial use. Mycologist, v.8, p.113-114, 1994. 